\title{
Synchronous Oligometastatic Non-Small Cell Lung Cancer and Isolated Renal Cell Carcinoma: A Case Report and Literature Review
}

\author{
Timothy K. Nguyen ${ }^{1}$, Alexander V. Louie ${ }^{1}$ \\ 1. Department of Radiation Oncology, London Regional Cancer Program, Western University, London, \\ Ontario, CA
}

$\square$ Corresponding author: Timothy K. Nguyen, timothy.nguyen@londonhospitals.ca Disclosures can be found in Additional Information at the end of the article

\section{Abstract}

A 58-year-old gentleman presenting with a progressive headache, visual disturbance, decreased appetite, and weight loss was found to have a localized clear cell carcinoma of the kidney and synchronous Stage IV non-small cell lung cancer with a solitary brain metastasis. This case illustrates the challenges in distinguishing between primary and metastatic disease in a patient with both renal cell carcinoma and lung cancer. We highlight the uncertainties in the diagnosis and management of this unique clinical scenario and the potential implications on prognosis.

Categories: Radiation Oncology, Oncology

Keywords: synchronous, non-small-cell lung cancer, renal cell carcinoma, metastatic, metastasis, oligometastasis, oligometastatic, concomitant, simultaneous, metastasectomy

\section{Introduction}

The oligometastatic state is a clinical paradigm whereby cancer patients with limited metastases (i.e., 1 to 5 ) that are amenable to aggressive management through resection or ablative therapy may survive beyond expectations [1-2]. At the time of diagnosis, non-small cell lung cancer (NSCLC) and renal cell carcinoma (RCC) present with metastatic disease in approximately $56 \%$ and $30 \%$ of cases, respectively [3]. Renal metastases have been reported in $16-23 \%$ of metastatic NSCLC cases while, for metastatic RCC, the lungs are the most common site of metastasis representing up to $45 \%$ of cases [4-5]. In situations with radiographic evidence of both pulmonary and renal tumors, the diagnostic challenge becomes differentiating

Received 09/30/2015 Review began 10/01/2015 Review ended 10/25/2015 Published 10/27/2015

\section{(C) Copyright 2015}

Nguyen et al. This is an open access article distributed under the terms of the Creative Commons Attribution License CC-BY 3.0., which permits unrestricted use, distribution, and reproduction in any medium, provided the original author and source are credited. between primary neoplasms and metastatic disease. We report a case of oligometastatic NSCLC with a solitary brain metastasis in the setting of a synchronous primary renal neoplasm. Informed patient consent was obtained prior to the drafting and submission of this report.

\section{Case Presentation}

\section{Initial assessment}

We present a case of a 58-year-old, right-handed gentleman with a medical history of Type II diabetes, obstructive sleep apnea, gastroesophageal reflux disease, asthma, gout, anxiety, and depression. He was an ex-smoker with a 40 pack-year smoking history. His oncologic family history was significant for his father dying from bladder cancer. 


\section{Cureus}

In the spring of 2014, the patient initially presented to his local emergency room with progressive frontotemporal headaches, a right visual field deficit, decreased appetite, weight loss, and a fall without sustaining traumatic injuries. On a review of systems, no respiratory, genitourinary, or additional neurological symptoms were described aside from increased daytime urinary frequency.

Physical examination revealed an obese gentleman (BMI of 41) with normal vital signs. The patient was alert, oriented to person, place and time, and ambulatory with a normal gait.

Cranial nerve exam revealed right visual field deficits. Auscultation of the lungs revealed clear, equal air entry bilaterally without any adventitious sounds appreciated. No masses,

organomegaly, or pelvic lymphadenopathy were appreciated on the abdominal exam, but this was limited due to his large body habitus. Digital rectal exam revealed a small, smooth, firm prostate without rectal masses.

A CT and MRI of the head was completed with the latter revealing a heterogeneous mass in the left occipital lobe with solid and cystic components measuring $2.9 \mathrm{~cm}$. There was associated cerebral edema with a $0.2 \mathrm{~cm}$ right-sided midline shift and no hydrocephalus identified (Figure 1).

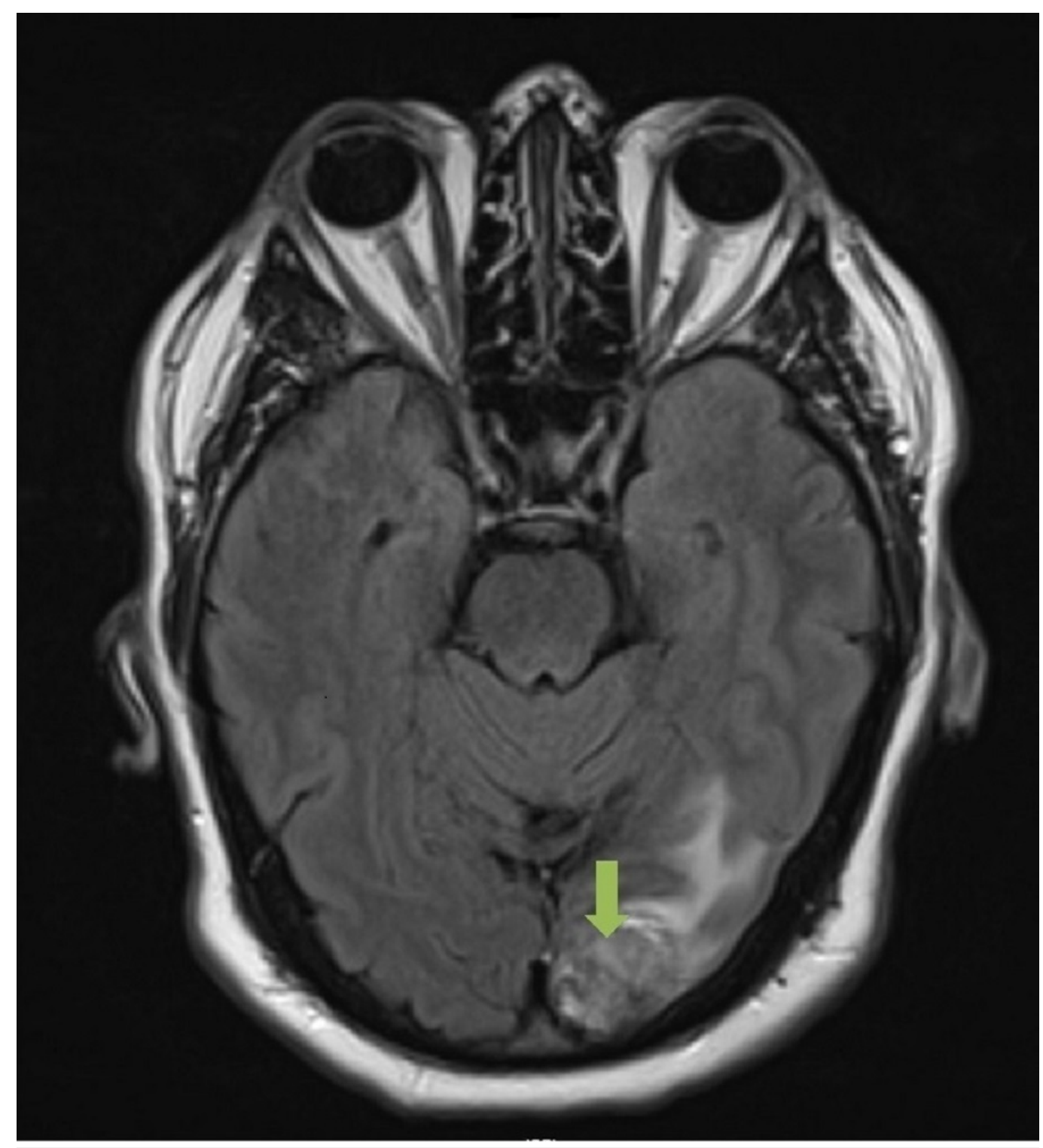

FIGURE 1: Preoperative MRI of the Head 
Axial slice. T2-weighted image that demonstrates a solitary $2.9 \mathrm{~cm}$ left occipital lobe adenocarincoma, metastatic from a lung primary.

\section{Initial management}

The leading differential diagnosis at the time was a primary brain malignancy, and accordingly, referral to neurosurgery at the same tertiary hospital was arranged. The patient subsequently underwent left occipital craniotomy and resection of the mass within five weeks of the initial referral. Pathology returned as metastatic adenocarcinoma, suggestive of a lung primary as it stained positive for cytokeratin 7 and TTF-1 and negative for cytokeratin 20. Adjuvant wholebrain radiotherapy was delivered to a dose of $30 \mathrm{~Gy}$ in 10 fractions, which was completed within six weeks from the time of surgery. The start date of radiotherapy was delayed by a couple of weeks as a result of a postoperative wound infection that was managed with oral antibiotics. During this time, the patient had a staging CT scan in the community encompassing the thorax, abdomen, and pelvis, which revealed a $5 \mathrm{~cm}$ left upper pole renal mass without other sites of disease. This report was not available in the patient's record; however, the dictated notes from the medical team indicated that metastatic renal cell carcinoma was the provisional diagnosis.

Concomitant with the planning and delivery of whole brain radiotherapy, the patient was also seen and assessed by the urology team at the same institution. Restaging CT imaging of the thorax, abdomen, and pelvis was completed two weeks after completion of radiotherapy, which revealed an exophytic solid renal mass arising from the upper pole of the left kidney measuring $4.9 \mathrm{~cm}$. In addition, there were now two other areas of concern: a $3.1 \mathrm{~cm}$ heterogeneous pancreatic mass containing cystic components and a $2.1 \mathrm{~cm}$ pulmonary nodule in the lower lobe of the right lung. Both lesions, radiographically, were thought to be metastases from a primary renal cell carcinoma. Considerations for management included cytoreductive surgery, up-front Sunitinib, or enrolling the patient on a clinical trial (Figures 2-3). 


\section{Cureus}

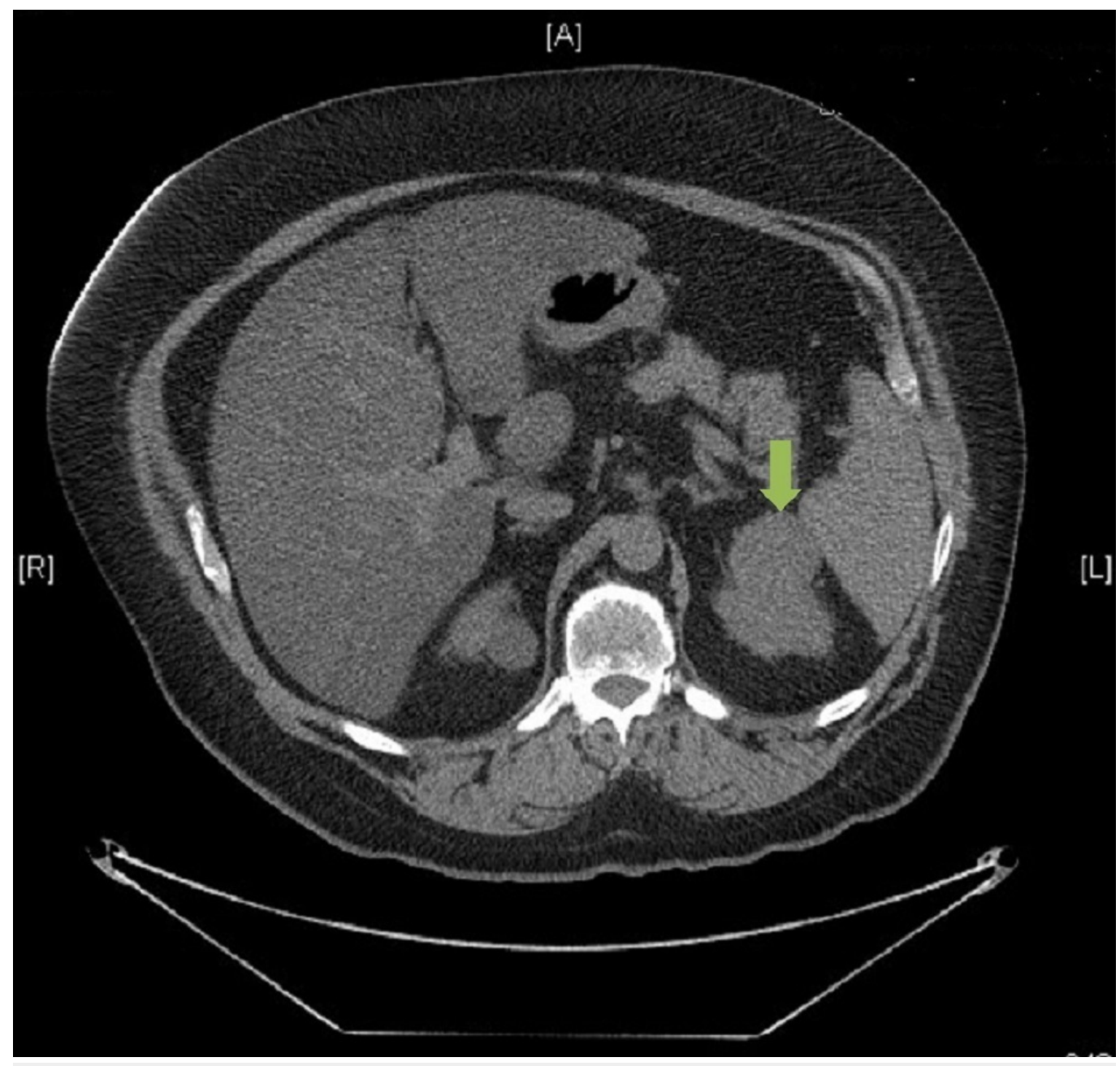

FIGURE 2: Preoperative CT of the Abdomen

Axial slice. Contrast-enhanced image that demonstrates a $4.9 \mathrm{~cm}$ isolated clear cell renal cell carcinoma involving the upper pole of the left kidney. 


\section{Cureus}

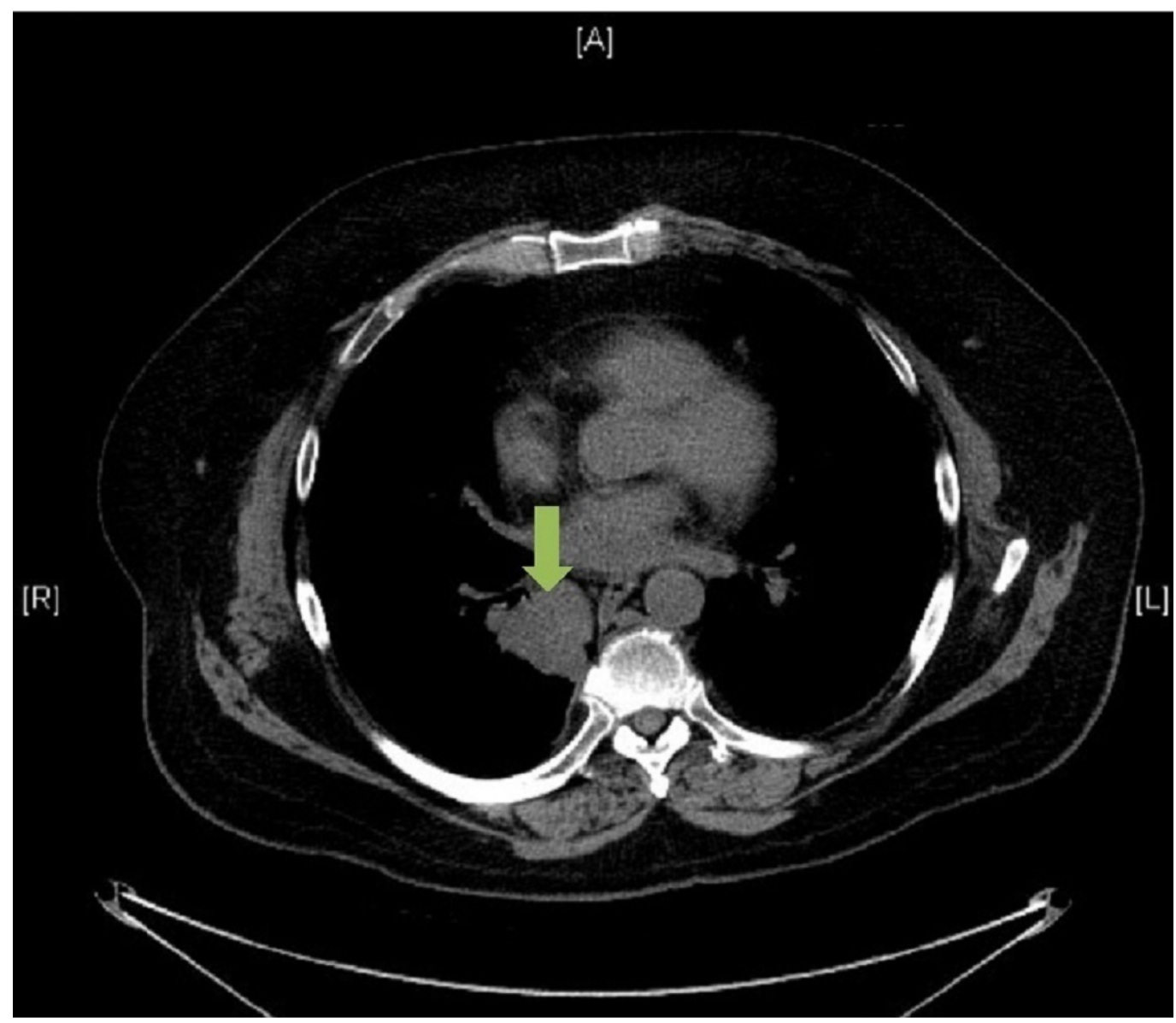

FIGURE 3: Preoperative CT of the Thorax

Axial slice. Contrast-enhanced image that demonstrates a $2.1 \mathrm{~cm}$ primary papillary adenocarcinoma of the right lower lobe of the right lung.

However, before the patient could return to further discuss management options, an MRI scan of the head, completed two months after finishing radiotherapy to assess treatment effect, showed evidence of an intracranial abscess in the previous operative bed. Specifically, there was an interval development of a $4.2 \mathrm{~cm}$ rim-enhancing fluid signal in the post-surgical bed. Urgently, the patient was brought to the operating room for a left occipital craniectomy for evacuation and resection of the abscess. The patient recovered well without any additional complications, although did not wish to seek any further medical care at his local institution.

\section{A second opinion}

The patient's primary care team in the community referred the patient to the multidisciplinary genitourinary oncology team at our center for a second opinion regarding management of the patient's remaining disease. Following a review of the original imaging and pathology reports, the possibility of metastatic lung cancer (given the immunohistochemistry of the resected brain lesion favoring a lung primary) or synchronous primary malignancies was proposed. A PET-CT scan was ordered, which re-demonstrated the pulmonary mass in the lower lobe of the right lung, now measuring $3.0 \mathrm{~cm}$ with an SUV uptake of 9 . A $1 \mathrm{~cm}$ non-18-FDG-avid subcarinal lymph node was also identified. The renal lesion measured $4.2 \mathrm{~cm}$ and was not 18-FDG-avid. A nodule in the pancreatic tail was thought to be a benign process, given its cystic appearance, stable size, and lack of 18-FDG uptake. 
The case was also presented at our thoracic multidisciplinary team conference with two recommendations provided. First, it was advised to complete the diagnostic workup, including biopsies of both the lung and renal masses, in addition to sampling the mediastinal lymph nodes. Second, depending on the results of the investigations, treatment should be considered in accordance with the oligometastatic paradigm, which could involve sequential resection of the pulmonary and renal tumors.

An ultrasound-guided biopsy of the kidney mass confirmed renal cell carcinoma (clear cell). The sample stained positive for CKAE1/AE3 and vimentin, while staining negative for CK7, CK20, and TTF-1. Through an endobronchial ultrasound (EBUS)-guided biopsy, tissue was obtained from the right lower lobe mass and a paraesophageal (level 8) lymph node. The lymph node was negative for malignancy while the biopsy from the mass showed the presence of neoplastic cells. Given the limited sample, pathologists were unable to distinguish definitively between a well-differentiated adenocarcinoma and a neuroendocrine tumor at that time. It was evident, however, that the lung and kidney pathology were distinctly different.

\section{Management}

In accordance with recommendations from both our multidisciplinary teams, the patient proceeded with radical intent treatment, first undergoing a video-assisted transthoracic surgery (VATS) right lower lobe lobectomy within two months of initial assessment at our center. Pathology revealed a unifocal tumor measuring $4.5 \mathrm{~cm}$ and was characterized as a Grade 2 papillary adenocarcinoma with negative margins. Eight lymph nodes were removed, and one peribronchial lymph node was found to be positive for malignancy with extranodal extension. Final lung pathologic staging was T2aN1.

Postoperatively, the patient met and discussed with the medical oncology team regarding the benefits of adjuvant chemotherapy in the setting of N1 and oligometastatic disease. Given the unclear benefit systemic therapy might provide for completely resected oligometastatic disease in a patient with moderate comorbidities, adjuvant chemotherapy was ultimately declined by the patient despite it being offered.

Restaging CT of the abdomen and pelvis was completed three months following VATS resection and demonstrated that the kidney mass had grown in size to $6.4 \mathrm{~cm}$ since previous imaging. The patient subsequently underwent a left partial nephrectomy three months later. Pathology revealed a unifocal $6.0 \mathrm{~cm}$ tumor in the upper pole of the left kidney, which was resected with negative margins. Histologically, the tumor was consistent with the previous biopsy, characterized as a clear cell renal cell carcinoma. Regional lymph nodes were not sampled. The final pathologic staging was pT1bNx, and no additional adjuvant treatment was felt to be indicated.

The patient was seen in follow-up three months following resection of the renal mass, which was 10 and 16 months from previous pulmonary and intracranial resections, respectively. He recovered well without surgical complications and was found to have no clinical evidence to suggest disease recurrence or progression. Repeat CT imaging of the head, thorax, abdomen, and pelvis revealed no evidence of recurrent or residual disease. Moving forward, the patient will continue with routine CT surveillance every three to six months.

\section{Discussion}


Synchronous primary malignancies are complex cases that present with diagnostic, prognostic, and therapeutic uncertainties, particularly if there has already been metastatic spread from one or both of the primary sites. We report on a case of oligometastatic adenocarcinoma of the lung with a solitary brain metastasis that presents synchronously with a primary clear cell renal cell carcinoma. The challenges surrounding this case stem from the propensity of lung cancer to metastasize to the kidneys and brain, and likewise, the ability for kidney cancers to metastasize to the lungs and brain. In such situations, histologic confirmation with immunohistochemistry can help distinguish between metastatic and primary disease. Given the paucity of evidencebased standards to guide treatment decisions in these clinical scenarios, we propose that such cases be discussed in the context of the relevant multidisciplinary tumor boards.

There have been a limited number of published cases reporting on the diagnostic and therapeutic approach to synchronous primary pulmonary and renal neoplasms. Similar to our case, a report from the Netherlands described a patient with a TTF-1 positive adenocarcinoma of the right lower lobe of the lung invading into the thoracic vertebrae that presented synchronously with an 18-FDG-negative lesion in the right kidney [6]. Initially, the case was considered to be Stage IV NSCLC with the renal mass representing a distant metastasis and, as such, was managed with an expectative approach. In the context of a second opinion and multidisciplinary reevaluation, however, a provisional diagnosis of two synchronous primary neoplasms was made. The lung cancer was managed with two cycles of neoadjuvant chemotherapy (cisplatin and pemetrexed) followed by surgical resection. The kidney cancer was managed with surveillance, given its stability in size, until it was later resected at the patient's request. The patient was disease-free at the six years following radical treatment, again highlighting the difficulty and importance in differentiating between primary versus metastatic disease.

Distinguishing between a renal metastasis of lung origin and a primary RCC (or vice versa) can be a difficult task. The use of PET imaging can be useful in identifying metastatic deposits in the kidneys, which tend to be 18-FDG-avid. However, a lack of hypermetabolic activity does not rule out the possibility of a primary RCC. PET imaging has been described to have a low sensitivity to detect primary renal malignancies, which was consistent with the experience of Mazouz, et al. and with our own findings in the present case [6-7]. Although renal metastases are typically bilateral and multi-focal, they can also present as a large, solitary metastasis in breast, lung, and colorectal patients [8]. Thus, obtaining pathology is advised in patients amenable for biopsy. In addition, only 1-3\% of patients with metastatic RCC present with a solitary metastasis [9]. When considered alongside additional supportive clinical evidence, this point may steer clinicians away from a diagnosis of metastatic RCC in cases of synchronous primary neoplasms with a solitary metastasis.

In a retrospective review of percutaneous core biopsies for renal masses, unnecessary surgery was avoided in $30 \%$ of cases in which biopsies revealed benign tumors or non-surgical metastatic disease [10]. Sanchez-Ortiz, et al. conducted a retrospective review of 100 patients with a renal mass in the setting of a non-renal primary malignancy. Fifty-nine percent of renal masses were malignant primary tumors, $19 \%$ were metastases (lung and lymphoma being the most common primary sites), $12 \%$ were benign primary tumors, and $10 \%$ had non-diagnostic biopsies. All cases of renal metastasis occurred in the setting of clinical progression or radiographic evidence of other metastases from the non-renal primary cancer [11]. The authors contend that for the majority of patients with a renal mass in the context of a clinically localized non-renal malignancy, the renal tumor is unlikely to be metastatic disease. Once pathology is obtained, immunohistochemical techniques can help direct diagnosis, with TTF-1 staining positive in approximately $76 \%$ of lung adenocarcinomas [12].

In the oligometastatic setting, there have been several publications that demonstrate a prolonged survival when pursuing metastasectomies in intracranial, hepatic, and pulmonary 
metastases [13-15]. However, randomized data to support a benefit in overall survival with radical treatment for oligometastatic disease has only been demonstrated in the solitary brain metastasis setting [16]. As the body of literature on the management of oligometastatic disease continues to grow, heightened attention towards its implications on prognosis and treatment will be vital in ensuring high-quality patient care.

\section{Conclusions}

In conclusion, we present a novel case of a 58-year-old gentleman presenting with a synchronous localized renal cell carcinoma in the setting of oligometastatic NSCLC with a solitary brain metastasis. In accordance with the oligometastatic paradigm, the patient is alive, disease-free, and well 14 months from the initiation of his treatment. Management of synchronous primary neoplasms, particularly in the oligometastatic setting, relies on a thorough diagnostic workup and careful consideration of all possible clinical scenarios, which should occur in the context of a multidisciplinary team approach.

\section{Additional Information}

\section{Disclosures}

Human subjects: Consent was obtained by all participants in this study. Conflicts of interest: In compliance with the ICMJE uniform disclosure form, all authors declare the following: Payment/services info: All authors have declared that no financial support was received from any organization for the submitted work. Financial relationships: All authors have declared that they have no financial relationships at present or within the previous three years with any organizations that might have an interest in the submitted work. Other relationships: All authors have declared that there are no other relationships or activities that could appear to have influenced the submitted work.

\section{References}

1. Hellman S, Weichselbaum R: Oligometastases. J Clin Oncol. 1995, 13:8-10.

2. Palma DA, Salama J, Lo S, Senan S, Treasure T, Govindan R, Weichselbaum R: The oligometastatic state - separating truth from wishful thinking. Nat Rev Clin Oncol. 2014, 11:549-57. 10.1038/nrclinonc.2014.96

3. Gay HA, Michalski JM: Cancer of the Kidney, Renal Pelvis and Ureter . Perez and Brady's Principles and Practice of Radiation Oncology 6th Edition. Halperin EC, Wazer DE, Perez CA, Brady LW (ed): Lippincott Williams \& Wilkins, Pennsylvania; 2013. 63:1245-1258.

4. Quint LE, Tummala S, Brisson LJ, Francis IR, Krupnick AS, Kazerooni EA, Iannettoni MD, Whyte RI, Orringer MB: Distribution of distant metastases from newly diagnosed non-small cell lung cancer. Ann Thorac Surg. 1996, 62:246-50. 10.1016/0003-4975(96)00220-2

5. Bianchi M, Sun M, Jeldres C, Shariat SF, Trinh QD, Briganti A, Tian Z, Schmitges J, Graefen M, Perrotte P, Menon M, Montorsi F, Karakiewicz PI: Distribution of metastatic sites in renal cell carcinoma: a population-based analysis. Ann Oncol. 2012, 23:973-80.

10.1093/annonc/mdr362

6. Mazouz A, Amaadour L, Souaf I, El Fatemi H, Amarti A, Erraisse MA, Oubelkacem E, Bouhafa T, Tahiri Y, Tazi MF, Mellas S, Arifi S, Mellas N: Synchronous malignant renal mass in patient with a lung cancer: case report and literature review. Pan Afr Med J. 2015, 20:22. 10.11604/pamj.2015.20.22.5541

7. Kang DE, White RL, Zuger JH, Sasser HC, Teigland CM: Clinical use of fluorodeoxyglucose F18 positron emission tomography for detection of renal cell carcinoma. J Urol. 2004, 171:1806-9. 10.1097/01.ju.0000120241.50061.e4

8. Choyke PL, White EM, Zeman RK, Jaffe MH, Clark LR: Renal metastases: clinicopathologic and radiologic correlation. Radiology. 1987, 162:359-63. 10.1148/radiology.162.2.3797648

9. Montie JE, Stewart BH, Straffon RA, Banowsky LH, Hewitt CB, Montague DK: The role of adjunctive nephrectomy in patients with metastatic renal cell carcinoma. J Urol. 1977, 


\section{Cureus}

$117: 272-75$.

10. Lebret T, Poulain JE, Molinie V, Herve JM, Denoux Y, Guth A, Scherrer A, Botto H:

Percutaneous core biopsy for renal masses: indications, accuracy and results . J Urol. 2007, 178:1184-88. 10.1016/j.juro.2007.05.155

11. Sánchez-Ortiz RF, Madsen LT, Bermejo CE, Wen S, Shen Y, Swanson DA, Wood CG: A renal mass in the setting of a nonrenal malignancy: when is a renal tumor biopsy appropriate?. Cancer. 2004, 101:2195-201. 10.1002/cncr.20638

12. Harlamert HA, Mira J, Bejarano PA, Baughman RP, Miller MA, Whitsett JA, Yassin R: Thyroid transcription factor- 1 and cytokeratins 7 and 20 in pulmonary and breast carcinoma. Acta Cytol. 1998, 42:1382-88. 10.1159/000332172

13. Patchell RA, Tibbs PA, Walsh JW, Dempsey RJ, Maruyama Y, Kryscio RJ, Markesbery WR, Macdonald JS, Young B: A randomized trial of surgery in the treatment of single metastases to the brain. N Engl J Med. 1990, 322:494-500. 10.1056/NEJM199002223220802

14. Morris EJ, Forman D, Thomas JD, Quirke P, Taylor EF, Fairley L, Cottier B, Poston G: Surgical management and outcomes of colorectal cancer liver metastases. Br J Surg. 2010, 97:1110-18. 10.1002/bjs.7032

15. Weichselbaum RR, Hellman S: Oigometastases revisted. Nat Rev Clin Oncol. 2011, 8:378-82. 10.1038/nrclinonc.2011.44

16. Andrews DW, Scott CB, Sperduto PW, Flanders AE, Gaspar LE, Schell MC, Werner-Wasik M, Demas W, Ryu J, Bahary JP, Souhami L, Rotman M, Mehta MP, Curran WJ Jr: Whole brain radiation therapy with or without stereotactic radiosurgery boost for patients with one to three brain metastases: phase III results of the RTOG 9508 randomised trial. Lancet. 2004, 363:1665-72. 10.1016/S0140-6736(04)16250-8 